\title{
Gardening: A metaphor for sustainability in information technology-technical support
}

\author{
Ellen Christiansen, Associate Professor \\ Dept. of Communications, University of Aalborg, \\ Langagervej 8, DK-9220 Aalborg Oest, Denmark \\ Email:ellen@hum.auc.dk
}

\begin{abstract}
Based on an empirical study of how technical support people work in a nonprofit research and consulting institute, the notion of gardening, first coined by Nardi, is unfolded as a metaphor for a sustainable way of making use of information technology (IT). Sustainability is defined as development characterized by optimal interaction in and between the resource systems and the economic and social systems.

Using Bateson's classification of interaction, symmetric and complementary, and of changes in interaction, feedback and calibration, a framework for understanding technical support as gardening is unfolded. Examples from a case study are used to illustrate how the technical support people in a specific organization, working as gardeners, play three different roles simultaneously: as experts (decision-makers, gatekeepers, creative planners) when working with the technology and wrapping up after productions; as colleagues, engaging in collaborative learning when working with users; and, when it comes to the outcome of joint efforts, they are like parents, taking pride in seeing the users walk away with the results even though their own contribution is invisible and maybe not even acknowledged. Furthermore, it is discussed how an organization can establish gardening relationships around technical support of the local design and use of IT.
\end{abstract}




\section{INTRODUCTION}

'Development that meets the needs of the present without compromising the ability of future generations to meet their own needs' WCED: 'Our Common Future'

This paper takes up the challenge of developing a conceptualization of what sustainable information technology (IT)-technical support may mean. Inspired by Gregory Bateson's philosophy, I assume that the distinction between environmental resources on the one side, and humans as mental systems intentionally misusing these resources on the other, is a false dichotomy. Nature is One ${ }^{1}$, but with a two-level structure: matter governed by embodied developmental principles. Carl Jung, following the Stoics, called matter pleroma and the governing principles, the mind, creatura. Bateson, in his epistemology (1958, 1979), built on these concepts together with the idea that the processes of interaction between different forms of matter are what cause the development of both. He categorized interaction in two types: symmetric and complementary interaction. The changes in interaction he saw as caused by differences in response, of which he found two types: feedback and calibration.

In Mind and Nature (1979), Bateson describes this typology of process as follows:

'I labeled the processes with the general term schismogenesis' [a term Bateson borrowed from Kretschmer, meaning 'first determining character within the individuals and beyond that creating intolerable stress'], and having put a label on the processes, I went to the classification of them. It became clear that a fundamental dichotomy was possible. The processes of interaction that shared the general potentiality of promoting schismogenesis were, in fact, classifiable into two great genera: the symmetric and the complementary. I applied the term symmetric to all those forms of interaction that could be described in terms of competition, rivalry, mutual emulation and so on (i.e., those in which A's action of a given kind would stimulate $B$ to action of the same kind, which in turn would stimulate A to further similar actions and so on. If A is engaged in boasting, this would stimulate $B$ to further boasting, and vice versa.) In contrast, I applied the term complementary to interaction sequences in which the actions of A and B were different but mutually fitted each other (e.g., dominance submission, exhibition-spectatorship, dependence-nurturance). I noted that these paired relationships could likewise be schismogenic (e.g. that dependency might promote nurturance, and vice versa)' (Bateson, 1979, pp. 191-92).

By putting these principles to work, side by side, Nature ensures survival of the fittest as well as (part of) the accidental deviation. Bateson suggested that a way to become conscious about Nature's way is through conscious reflection on 'the pattern that connects' levels of process and form and accordingly re-orientation of action-patterns. This kind of reflection-in-action he labelled deutero-learning, a concept which relates to the term double loop learning or learning to learn. 
I take sustainability in a Batesonian sense to be in line with the WCEDdefinition of sustainability quoted initially. In Mind and Nature, Bateson remarks:

'It seems that we must ask: What characterizes those adaptations that turn out to be disastrous, and how do these differ from those that seem to be benign and, like the crab's claw, remain benign through geological ages? The question is pressing and relevant to the contemporary dilemmas of our own civilization. In Darwin's day, every invention appeared benign, but that is not so today. Sophisticated eyes in the twentieth century will view every invention askance and will doubt that blind stochastic processes always work together for good. We badly need a science that will analyze this whole matter of adaptationaddiction at all levels. Ecology is perhaps the beginning of such a science, although ecologists are still far from telling us how to get out of an atomic armaments race. In principle, neither random genetic change accompanied by natural selection nor random processes of trial and error in thought accompanied by selective reinforcement will necessarily work for the good of either species or individual. And at the social level, it is still not clear that the inventions and stratagems which are rewarded in the individual necessarily have survival value for the society, nor vice versa, do the policies that representatives of society might prefer necessarily have survival value for the individuals' (Bateson, 1979, pp. 173-74).

Even though we know all too little to be sure, it might be worth trying to characterize optimal levels of interaction, the most sustainable ways of interaction, in order to apply the concept of sustainability to policy-making and planning. As suggested by Barbier (1989), the concept must be made operational and applicable to all forms of economic and social activity in a more concise, systematic and rigorous way. So, when I try to put Bateson's and others' ideas to work by analogy using the gardener-metaphor, I have to admit the intuitive character of my project, as when I suggest parallelling the symmetric and the complementary type of interaction with the different ways a gardener cultivates, nurtures, harvests, and composts her material. However, the case study (Christiansen, 1996b) from which I report here, came into being because I witnessed on a daily basis for quite some time how technical support was done in this organization, and I found that what I saw was actually an instance of a mixture of symmetric and complementary interaction that deserved the label 'good gardening' and that might serve as an example of sustainable conduct.

Using the gardener metaphor for the design and use of IT is not my idea. Bonnie Nardi, in her wonderful book on end-user programming (1993) coined it when she pointed to the gigantic potential that may be found in facilitating enduser computing by employing 'super users' as local gardeners. Nardi (1993) uses gardener as a metaphor for a super user of a certain kind: a person who

- is responsible for nurturing fellow employees and providing strong support so that employees can perform as effectively as possible

- is coming from the rank and file: who knows the domain, the users, the frustrations and problems

- has a genuine interest and enjoyment in assisting less knowledgeable users on the one hand and, on the other hand, has a desire to learn the way around an operating system and a programming language

- has great communication skills 
- is trusted by all parties

- provides an important bridge between systems administrators on the one side and domain experts on the other, and can also communicate with users

- makes managers feel confident that the standardization and integrity of the macros, programs and data used by their staffs will be maintained

- is discovering and bringing to the group a new productivity tool that would not have been found by the average user just trying to get work done

- makes a special effort to keep up with advances in the field and to be cognizant of the changing work practices in which s/he is not directly participating ${ }^{2}$.

I am inspired by Nardi's examples and application of the metaphor of gardening but have extended it to apply to dedicated technical support.

\section{AN ETHNOGRAPHIC STUDY OF HOW IT-TECHNICAL SUPPORT PEOPLE WORK}

Encouraged by the correspondence between what I witnessed daily and what I had learned from Nardi, I set out to do a field study on the work of two technical support people, the systems administrator and the audio-visual support person ('the video-maker') in a small, nonprofit research and consulting institute. The institute, founded in 1986, has a yearly revenue of about 4 million US dollars and employs about 55 research scientists, staff, and consultants. At the time the study was carried out, a total of 124 computers (57 on site, 11 off site, 7 servers and the rest distributed in schools and other places) were serviced by the systems administrator. When the systems administrator went on trips or vacation, her job was covered by the video-maker. Video is used in most of the research and consultancy work done at the institute: data from school or workplace settings are videotaped, analysis is done by detailed examination of the video tapes, and video is used as a medium for communicating the findings to clients and scientific communities and as a medium for instruction. The video-maker puts up cameras, shoots the videos and edits presentations and tutorial videos in collaboration with researchers. Apart from the ongoing support for data collection and analysis, in 1995 ten videos were produced ranging from 7 to 25 minutes. Since the field study began, additional support personnel have been hired for the systems administrator as well as for the video-maker.

The data for this case study consist of 24 hours of observation, 4 hours of videotaped video editing, and 5 hours of videotaped interviews and videotaped focus group discussions. The results have been thoroughly discussed with the individuals studied in order to clear up misunderstandings and get their full consent regarding the use of the data. Clearly, the people I studied were not endusers in the sense defined by Nardi but people whose jobs it is to make information technology a valuable contribution to products, in this case, teaching and training material, reports, presentations, papers. Then, what did I see?

In addition to the communicative skills regarding translating from the technical world to the world of applications and being responsive to actual needs and levels of competence emphasized by Nardi, I found that skills for physical installation and repair, in sum for coping with the physical world, were crucial. This physicality showed up as a practical ability, an inclination to nurse the 
technology, monitor the conditions, diagnose repair, and reuse equipment: as a caring and playful attitude towards the machinery.

Also, it seemed important that the bridging position, mentioned by Nardi, between management and employees comprised bridging across the different communities of practices within the institute. Because of the knowledge about everyone's needs and wishes gained through bridging, the support people seemed to operate from a notion of a common good with respect to the use of technology with, it seemed, the acceptance of the people in the organization to take action accordingly.

Unlike the gardeners profiled by Nardi, the technical support people I met did not come from the rank and file of those they were supporting. They were hired as technical support persons. Both had, when we first met, an academic degree as their educational background, several years of experience from other companies, plus 3-4 years of experience at the institute. Both also had a personal professional network outside the organization, the systems administrator with technical support people and people in the software industry, and the video-maker with other videomakers, mostly freelancers. Compared to what I have seen in similar organizations, these two support people got a lot of work done and maintained quite a high technical standard on a low budget. Because of this, and because people at all levels in the organization frequently expressed gratitude for what they did, I assume their work was successful from the point of view of the organization. My impression from being around the institute for a year was that of a growing technical competence among people in the organization which I, more than from their actual teaching, ascribe to the way in which they created a safe atmosphere for people's experiments and for peer learning.

To give an impression of what their way of working was like, I specify the metaphor of gardening to cover four distinct areas of technical support work:

- cultivation of the technical equipment, programs and infrastructure

- nursing users, the peer learning through which the technical support of the production is accomplished

- harvesting, presenting high quality products of which the technical support is an important, yet transparent, feature

- composting, making sure that byproducts of the production processes, and parts of all kinds are kept for potential re-use.

\section{Cultivating ...}

As far as the cultivation of equipment is concerned, immediate and cost-effective repair is important. To obtain this goal, good formal and informal relationships to a network of vendors seemed crucial. Another important parameter was the ability to make informed choices balanced by an overall vision and strategy for expansion of the IT use. The following observation from the study illustrates this.

'... The computer at the reception desk has crashed. While the receptionist is away for a meeting, the systems administrator sits at the reception in the receptionist's chair, drinking soda, rebuilding the desktop of the computer and meanwhile taking care of the reception. She is fixing the machine with Norton Disk Doctor, operated through her little self-made portable work bench, which includes an external hard drive on which Norton is available. This hard drive 
has become her most precious and handy repair tool. It is very good for fixing high level problems on the operating system. Application breakdowns are usually taken care of by the application itself. For the rest, it is in the long run cheaper to take them to the repair shop, where all replacement parts come free from the vendor. Actually, it has been on her mind to become licensed herself in order to get the parts for free, but you have to be a retailer to get them for free, so it wouldn't work. Instead she tries to bargain and get the prices down as much as possible. This machine crashed because the receptionist kept rebooting it several times, which may have caused a good deal of damage.'

'One of the researchers passes by. Seeing her makes the systems administrator remember that she has a message for her, 'Oh, I just had a request from somebody about groupware and organizational learning. I referred him to you.' They engage in a conversation that, after a while, turns to Lotus Notes. Together they develop an idea about how to make really functional integration between platforms over/via the web. The researcher leaves, and Norton has succeeded so far that the systems administrator feels inclined to do a little clean up of the desktop icons. It is a matter of aesthetics, but in the flow she gets rid of some big chunks of irrelevant software. Since the reason the machine crashed was basically overload the clean up has a functional aspect, too. It crosses her mind that partly the problem has risen because she did not do the best job when installing this machine. She does a reboot to see if the machine is OK. Now another colleague comes by. She has a problem with her name popping up on the receptionist's computer. While the system administrator tries to pay attention to this problem, somebody is on the phone asking about a video conference for the board meeting on Friday with a test-run on Wednesday, of which she is in charge. She talks and takes notes, while still nursing Norton. Then she puts the guy on the phone on hold in order to go and get an email message from the video-maker (who is out of town this week) that will explain the details. While rising, she puts Norton on a new task. Back with a printout of the video-maker's message she shifts the phone to the other ear so that she can operate the mouse simultaneously. The accountant passes by with a message, and she nods. Together with the person on the phone, she goes over a scenario as to what it should be like on Friday. After this conversation, she turns back to the computer for a moment and then unpacks some new motherboards that just arrived in the mail. She is careful preserving the boxes and other wrapping material because she knows that she'll need them next time she is going to send some stuff off safely for repair. Then she shifts mode again, now listening and feeling if the computer seems up and well. The accountant comes by again and talks about some more paperwork. While waiting for Norton, she talks with the receptionist who has returned from her meeting. The receptionist explains how the machine just went berserk, and she did a reboot, and a reboot and another reboot. The system administrator takes the opportunity to emphasize the importance of never doing more than one reboot ..

According to this fairly accurate account, the work with the disk doctor tool, the phone, and interaction between colleagues takes place simultaneously. The systems administrator restores a disk, does some teaching that should prevent further accidents, makes an appointment about conference support, exchanges 
information about forthcoming software investments, unpacks some new equipment, stores the wrappings and takes care of some paperwork in less than one hour.

\section{Nursing ...}

When it comes to nursing, that is to say, peer learning, Nardi points out that the skills for this are a special gift:

'... A person who is technically skilled but uninterested in intensive interpersonal interaction may not have much of a green thumb when it comes to helping other users. By the same token, a person without sufficient technical skill would not be an effective gardener ...' (Nardi, 1993, pp. 117-18).

But even the most responsive gardener cannot make sure all by herself that the users in the organization who need technical support get what they need to produce the best possible products. Time would not allow her to support each and every user when needed. What can be done, and what the support people I studied did, is always to be attractive role models in their behaviour, and thereby put up an umbrella of inspiration under which the users can experiment safely, and safeguard experiments by being immediately accessible as firefighters if something gets out of hand.

'You try to make them feel good about whatever it is they try to learn. And give them the pros of a piece of software as opposed to the negatives, so you just try to make them feel good about what they are doing, and then hopefully it works out and then they go out and they learn and they come back and they can teach us actually! It happens. They actually learn something and they can say to you, 'You can do this,' and then we go, 'Oh I didn't know that,' and so ... For instance a colleague taught herself Illustrator and you know now, when people want to learn Illustrator, we just refer them to her.'

But quite often nursing and cultivation does not come out as nice and easy as that. An example where one of the senior researchers has asked for help to learn how to receive and send faxes via modem when working from home may illustrate a far more typical condition.

'This guy is always busy so the technical support person is not frustrated when the meeting is re-scheduled a couple of times, and she is also prepared for interruptions during the planned one-hour session. She even tries to take advantage of getting his attention by bringing her proposal for next year's budget, since he is responsible for the budget and this might be her only chance to give him an informal introduction to her line of thought. In advance, she has made a few small stickers to put on his computer with short cuts for what he needs to do to get his faxes through. He is working on his computer when she arrives. Somehow the conversation starts out on a question about how to rearrange address books in the email software. He has scenarios prepared, and he operates the mouse. She responds to his scenarios and explains the possibilities. During this, he gets a lot of small arrangement problems cleared up. She tries to stop him and make him reflect and learn. They get into discussion about the procedure of organizing mailing. She tries to use the screen as a prompt of what to talk about concerning mailing. Gently she turns the conversation towards today's issue of faxing via modem. She suggests 
doing an actual test and begins to explain the basic principles and to fix the telephone so as to take the example all the way through. He protests, he wants to move on since he has more problems, but now she is insisting. It takes some time for the fax to get through, and meanwhile he asks, 'How do I know, the document is send,' and grabs the mouse to demonstrate his problem. Doing this reminds him that he does not know the difference between single click/double click. She explains both the clicks and also about the word-install names, then the machine crashes. This tends to happen when this specific text editor runs together with the mail system. She has to get the machine up again. She takes the opportunity to announce, that in a short time she will be releasing a field researchers' survival kit which will help telecommuters, for instance, to build up their machine when it crashes. Now he asks how to fax. So at that point the planned session starts, 45 minutes after the meeting began. He talks about the fax and she explains. Then they turn to talk about HP fax, and talk prices. His machine is still working to get up again. She returns to the issue of crashes. He asks, 'Can you teach me how to build up my desk top?' And he continues talking about a new monitor he wants, and goes from there to the budget, while she seeks to establish a definition of what it means when a computer crashes. She goes and gets the Norton utilities, in order to instruct him about that. While she is away, he reads through the draft of the computer budget. From now on the conversation follows two parallel tracks: the budget and the issue of rebuilding a desktop. It takes until his secretary comes and takes him to his next appointment.'

\section{Harvesting $\ldots$}

A third aspect of gardening is harvesting, that is to say presenting high quality products of which the technical support is an important, yet transparent, feature. How this works is illustrated in the following example, where the video-maker tells me about his work:

'When I cooperate with people around editing, what we basically do: we take the analog videotape and digitize it. When they bring me the tape, then I automatically sit there and I am editing it in my head while I am looking at it. OK, so what I have the researcher do or whoever I am working with, they sit down and they identify the clips they want to use, and since there is a limited amount of hard drive space on this machine, it can only store up to like an hour of video and two hours of audio, so what I do is I have them identify the clips they want to do, then I digitize them, put them on a track sheet to edit, and then at that point I have the person that I am dealing with come in and sit down and then we decide, you know, is this really the clip you wanna use, and what kind of transition you want to do, and then if we are gonna do voice overs or any sort of graphics, you know that we are going to put in between the clips, that's when we decide or that's when I have that person decide ... That is my background as a film and TV major: I kind of can get a sense of what they want and then the best way to show people what they want to show them, you know, with the videotape that we have collected. That is the best part of my job, that is what I was trained to do.' 
'... What if they do not want to collaborate, and just want me to do it? Well, those rare cases, where people say, 'Just go ahead and do it!', that, of course, gives me the creative freedom. But the part I don't like about that is, when they say, 'I don't know, you just do it!' And then I do it and they don't like it, you know, and it is, 'Well, why didn't you say something then?'

'... What do I do if their ideas seems kind of odd? Well, then I try to explain to them why that won't work, you know, and I'll say: 'That's a great idea, but you know, this probably would work better, you know. Try it this way,' because they'll come up with these ideas and these ways they want to do things and sometimes it is not feasible, you know, for one thing or, if it is possible, it is going to cost a lot of money to reproduce it and that sort of thing ... But with this new video editing system I have got with the cube, I haven't had any problems with having to say no to people on their ideas.'

'... And then it is funny, because then, after we do the video tape, they'll take it and they'll look at it for a few times and they'll look at it with other people and then they'll come back and say, 'You know what: maybe we can improve on it with this,' and then I'll go, 'Yeah, that is true, we can cut that out and we can add this and do this in stead of that,' so they are learning as well.'

'... I have given them a sort of a guideline, in the sense that - actually mostly it is by repetition - if there has been one particular group that has done more than one video, then after the second time then they kind of get a sense of what I and the machine can do, what they want, and then if there is something else that they think about that they might want, then they'll say, 'Well you know, can we do it this way?' And, more often than not, we can do it that way and then we just improve on it in that way. So they are learning as well and they are learning to step outside of that boundary, that guideline sort of thing ... [Then] it becomes more fun, because they are more knowledgeable and then they, we kind of feed each other, and then they'll come up with an idea and then I go, 'Oh yeah, that will be neat, and then, plus we can do this on top of it,' and so it's a nice interaction there.'

When shadowing the support people I was somewhat puzzled by seeing them with a smile say good-bye and good luck to researchers who went off to a client with a presentation that was the result of maybe several days of intense cooperation between researcher and support person. Of course it is part of their job. But what kind of a professionalism is it that accepts and maybe even enjoys to see others receive the applause for what has been accomplished in collaboration? Maybe a label like 'back stage professionalism' would be appropriate. At least, the support staff I talked to, said that they like to work back stage, 'to be behind the camera, not in front if it.' They seemed to prefer a parental role, like when children run out into the garden, eat all the peas or plums they can, and continue their play while their parents watch them and, even with a happy smile, clean up the mess they left. If there is going to be another harvest, there must be someone who cleans up and puts things aside. But as a career trajectory? I kept being puzzled until I overheard some conversations between the technical support staff and their peers outside the organization and heard them boast of some of their accomplishments. Then I understood that they, too, had an audience, a peer group with whom they compete and from whom they get admiration. 


\section{Composting ...}

Key concepts in this aspect of the work are the notion of common good and an overall strategy: acknowledging that users are individuals with individual personalities, that they deserve respect, but that the only one able to build a bridge between the users and the concern for the common good is the person who knows the needs on both sides as well as the actual options, and takes a professional pride in striving to get the best quality solutions at the cheapest price.

What in this case made most ends meet was a consistent recycling behaviour on the part of the gardeners. One of the things I learned first was that a computer would not arrive on my desk as a total solution. Computers consist of different parts, and a solution means a combination of whatever spare parts are available and are working at the moment. I could go to the support staff and express an urgent need for a better headset, a mousepad, a printer, a monitor, a hard drive, and I would find them look around the immediate workspace then, if nothing was found there, go through a range of replacements until I got my thing, typically from someone who got a more adequate replacement by having a spare part from someone else who ... and so on.

The never-ending story of preserving, storing and reusing seems more than anything to be the 'fertilizer of the soil'. Always fill up printers with paper when passing by, always tidy up in the server room, always keep boxes and paper when unpacking so that stuff can be sent safely to repair, enjoy unpacking, checking and nursing hardware as well as software, always put on a happy face when asked for the same explanation over and over again: that is part of a never-ending story of keeping a technical support garden.

\section{NO GARDENERS WITHOUT A GARDEN}

You cannot work as a gardener unless the organization where you work allows you. When it comes to the implementation of a gardening attitude, the bottom line is the organization's actual cost-benefit assessment. I have only one organizational context to report from which is justified by the truly exploratory state of the idea, but which is, of course, still a limitation. The example given may give rise to more questions than it answers. But at least in this case, neither the management nor the users would like to be without technical support staff working with a gardening attitude even though, for the management, it meant delegation of control and even though, for the users, it meant that the gardeners' concern for the common good could prevent them from having their sweetest technological dreams come true.

The organization studied is young and enthusiastic, and rich on personal relationships across communities of practice. The technical support people are located in the office space, in cubicles similar to and next to those they are supporting. Learning is a basic value of this organization, implicitly as well as explicitly. Learning as an implicit value expresses itself in the non-hierarchical structure and in the open and informal way of communicating that allows everyone to ask for help because it is acknowledged that everyone is an expert with regard to certain aspects of the work. Accomplishments of learning are recognized and 
cheered. At the more explicit and formal level, quarterly, biannual and annually meetings are milestones and reference points and opportunities for coordinated reflection with respect to different aspects of the organization's activity. At these meetings, everyone participates in discussions about what has been accomplished and what should be learned and in which direction to expand. Organizational memory is a research topic for some, but certainly a boundary object for everyone.

On the economic side, expenses related to IT fall into three categories: a) investments and maintenance of network, machinery and software, b) teaching and learning how to use it, and c) productions. The use of computers and video, of the Internet and intranet, is crucial to the work at all levels in the organization. And even though the budget is limited, all three categories of IT expenses are present in the budget and are subject to the gardeners' constant efforts to balance the budget and to make sure that the productions are the raison d'être of the investments, and that the teaching and learning effort is made timely so as to spin off in productions. This balancing takes a specific form in each category: with respect to investments, the gardeners repair and reuse as much as possible in-house, and they do as much of the cabling, cleaning, and the soldering as they possibly can themselves. The teaching primarily takes the form of peer learning supported by firefighting when necessary, aimed at creating as many teachers and learners as possible. When it comes to the production of documents and videos, the gardeners are constantly trying to help people pay attention to waste problems, avoid unnecessary printouts, and support whatever can be done to reuse pieces so that the investment in production time is balanced by the scope of the use.

Although this organization has its collectors and those who always need the hottest software, even these people seem to accept that their needs are adjusted to the overall long-term plan for expansion, and that they will get spare parts and reused products if those solutions meet the need they have expressed. And those in the organization who are most reluctant to engage in and benefit from the IT wonder world are tempted and dragged and tempted again, but never forced against their will. The email system is used extensively to communicate news, events and problems, which makes the IT situation a dear and ready-to-hand subject of conversation, just like the weather in more unstable climate zones. Affections of all kinds can be expressed by blaming it on the technology, and the technology may even sometimes be the carrier of hope, like 'once we get on to the next version of Netscape ...'

Now, it was not exactly the Garden of Eden I studied. But this organization had a remarkably successful fit between the organizational culture, the willingness and determination of management to delegate power and authority, and the responsibility to create and develop a notion of the common IT good deliberately taken up by the gardeners. By taking a position as middle-men, they did reverse the tendency that those who were already well off with equipment and service got more because they were able to express more advanced needs. By nursing and developing the most reluctant users and preventing the most anxious from eating up all the cake just for the sake of personal appetite, they supported a balanced growth.

Along a similar vein, by acknowledging and creating pride in the entire organization, these gardeners as well as other employees created mutual relationships with the community and the neighbourhood: outdated equipment was 
given to schools and charity organizations, and people from the outside were invited to see, to try out, and learn about IT possibilities. And they arranged that the organization hosted exhibitions of artwork from various groups in the community - a gesture of grace, one could say, but a gesture that did a lot of good for the culture inside the organization because, in a very concrete way, it symbolized that 'we are committed to the surrounding community and we care.'

But learning implies change, and change may be experienced as unpleasant or even painful. Situated learning in today's workplaces is also learning under stress since things tend to run behind schedule. For example finishing off products, in terms of layout and final editing, is always last-minute-and-should-have-been-done yesterday stuff, and the necessary extra time for learning is usually not built into such time frames. Even in this organization, those in charge of the administration, layout, and the finishing of products tend to feel that the researchers do not take these parts of the work seriously enough. But unlike most organizations, people believe that pointing to these issues may bring about change for the better. For instance, the administration staff decided on an arrangement, a one-day workshop called 'Take your researcher to work'. The agenda was 'to increase our awareness of what it takes to get a job done.' The format was that the administration staff would put the researchers to work with some of the stuff usually taken care of by themselves to give the researchers hands-on experience and thereby to develop a shared understanding.

All in all, the responsive attitude on the side of the organization did match the situatedness ${ }^{3}$ and the responsiveness to immediate needs, shown by the gardeners.

\section{SUMMARY AND CONCLUSIONS}

My motive throughout this paper has been to give some flesh and blood to a conceptualization of sustainability within the field of IT-technical support. I have proceeded by borrowing a couple of concepts from the philosophy of Gregory Bateson, and by extending Nardi's metaphorical use of the concept of gardening to IT-technical support. Over two sections, I have presented an example of giving ITtechnical support with a gardening attitude. Now let me return to my point of departure: gardening as a metaphor for a sustainable way of conducting IT-technical support. What is it about gardening that supports the principles of sustainability?

In the introductory section I suggested sustainability is synonymous with nondisastrous or benign evolution: an evolution that manages to be adaptive without turning addictive. Bateson himself was very cautious and stated that 'We lack any systematic knowledge of the dynamics of these processes' (Bateson, 1979, pp. 174) [the processes of interaction that result in either adaptation or addiction]. In line with Bardier, I suggest that sustainable development is the optimal level of interaction of three systems: the biological and resource system, the economic system, and the social system through a dynamic and adaptive process of trade offs (Barbier, 1989, pp. 184-85). An 'optimal level of interaction' is characterized by what Bateson calls adaptive evolution, a composition of symmetric and complementary forms of interaction, adjusted by calibration and feedback. In those cases when the interaction becomes subject to reflection, it may be possible to engage in what Bateson called deutero learning, a communication about what 
connects different patterns of interaction, which in turn may lead to interactions of a new type. For example, when I asked the gardeners in my case study about how they encouraged users to learn about new technology, they immediately reframed my question by saying: the question is rather do we ever stop people from learning? They interpreted my understanding of learning as synonymous with teaching, compared this to their own understanding of learning as ongoing practice, then came up with a pattern they thought connected these two conceptualizations of learning and therefore would be a relevant outset for answering the question. This points to an important area of further investigation, namely the connection between sustainable development and deutero learning, and between deutero learning and the, nowadays so much demanded, double loop learning.

With examples from the case study, I pointed out four distinctive aspects of gardening

- cultivation of the soil, that is to say the technical equipment, programs and infrastructure

- nursing of plants, that is to say peer learning through which the technical support of the production is accomplished

- harvesting the fruits, that is to say presenting high quality products of which the technical support is an important, yet transparent, feature

- composting the remains, that is reusing all useful ideas, byproducts, procedures and technical components.

The cultivation of the soil, I found was characterized by the strongest possible degree of recycling, internally and in exchange with the community, but conducted by the gardeners as the experts, taking upon themselves to supersede rivalry between different, more or less resourceful, people while showing concern for the common good. Nursing the users was done on the gardeners' initiative by having any interaction turn into peer learning, so that the gardeners were the more capable peers with respect to the technical and, to a certain degree, the aesthetic aspects, while the users were more capable with respect to content so that they got what the video-maker called 'a nice interaction going on there'. When harvesting the fruits, the users would be acknowledged fully for the reports, videos, and so on by their clients, while the gardeners would be bystanders like parents. But the gardeners would be acknowledged by their colleagues in the profession outside the institute, and also by the community inside. And, in the flow of wrapping up after a production, the gardeners would be experts in composting the remains, so that all reusable parts of the process would be kept safe in order to fertilize the soil further.

In all aspects of gardening, I have found examples of both symmetric and complementary interaction. The interaction between gardeners and users is, with respect to cultivation, symmetrical in the sense that they compared themselves to other colleagues in the field of technical support in seeking to be the best. Their interaction with the machinery is complementary in the sense that they listen, they are supportive, and they talk about the computers as 'puppies' or even 'babies'. When it comes to people, the nursing, the nature of the interactions becomes complementary: 'We kind of feed each other' is the video-maker's phrase. It is primarily a human-human interaction, the machinery is a medium, something that is supposed to work transparently without breaking down and, when it eventually breaks down, the interaction becomes human-machine and becomes symmetrical, like a competition, where users usually lean back and give in while the technical 
support people lean forward and fight. Harvesting the fruits is characterized by complementary interaction between gardeners and users, like between parents and children, and the gardeners outsource their symmetrical interaction with gardeners elsewhere. Composting the remains is, by definition, a complementary endeavour since it is basically a matter of supply. But watching the gardeners in the process revealed the impression that at the same time they were also engaged in a competition with themselves to see how creative they could be in making sure that nothing went to waste.

The hypothesis I have tried to unfold here, by now in an absolutely explorative form, suggests that technical support could be characterized as sustainable to the extent that both symmetric and complementary forms of interaction are at play in all aspects of their work: cultivating the soil, nursing the plants, harvesting the fruits, and composting the remains. The framework would, when researched more thoroughly, offer a foundation for discussion about sustainable IT-technical support.

\section{ACKNOWLEDGEMENTS}

I am grateful to Phil Draper, Ann Mathison, and their colleagues at the Institute for Research on Learning, Menlo Park, California for having introduced me to ITsupport as gardening, and I owe Bonnie Nardi a lot for having brought my attention to the important qualities she addresses under the heading of gardener. Helga Wild (1996) and Libby Bishop have provided ideas and fruitful criticism while I struggled with writing the report. Unknown referees have provided me with encouraging criticism on a former draft of this paper for which I am very grateful. My husband, John Thogersen, has spent hours discussing my ideas, and drawing on and offering me his insights on sustainable consumer behaviour.

\section{REFERENCES}

Barbier, E.: Economics, Natural-Resources, Scarcity, and Development. Earth Scan, 1989

Bateson, G.: Naven. Stanford University Press, 1958

Bateson, G.: Mind and Nature. A Necessary Unity. E.P. Dutton, 1979

Bjerkness, G. et al. (eds.): Organizational Competence in System Development, Lund, Studentlitteratur, 1989

Bjerkness, Gro and Tone Bratteteig: The Application Perspective - An Other Way of Conceiving EDP-based Systems and Systems Development, in Săaksjărvi (ed.): Report of the Seventh Scandinavian Research Seminar on Systemeering, Helsinki, 1984

Bødker et. al:: The AT project. DAIMI PB-454. Aarhus University, 1993

Christiansen, E. (a): Tamed by a rose in: Nardi, B. (ed.): Context and Consciousness. MIT Press 1996

Christiansen, E. (b): A Gardening Attitude. Technical Report. The Institute of Research on Learning, Menlo Park, California, 1996

DeDijn, H.D. (ed.): The Way to Wisdom. Purdue University Research Foundation, 1996

Greenbaum, J. and M. Kyng: Design at Work. Lawrence Erlbaum 1991

Josefson, I. (ed.): Spraak och erfarenhet (Language and Experience). Carlssons, Stockholm 1985

Lave, J. and E. Wenger: Situated Learning. Cambridge University Press, 1991

Nardi, B.: A Small Matter of Programming. The MIT Press, 1993

Suchman, Lucy A.: Plans and Situated Actions. The Problem of Human-Machine Communication. Cambridge University Press, 1987 
Thoresen, Kari: Principles in Practice: Two Cases of Situated Participatory Design in D. Schuler and A. Namioka (Eds.): Participatory Design. Lawrence Erlbaum Ass., 1993

Wild, H.: Distributed teams, mobile workforce, distant education, remote work: Are we living on credit? Paper presented at EDRA 1996, June 1-2, 1996, Salt Lake City, Utah, USA

(WCED) World Commission on Environment and Development: Our Common Future. Oxford University Press, 1987

1 For a further elaboration, it might be relevant to take up Spinoza's argumentation on the oneness of nature and his reflection on nature (DeDijn, 1996).

2 Another word for these qualities could be 'caring rationality'. In Scandinavia, researchers have for decades conducted research on computing in organizations from an end-user perspective (Bjerkness, G. et al., 1989; Bjerkness \& Bratteteig, 1994; Bødker et. al., 1993; Christiansen, 1996a; Greenbaum \& Kyng, 1991; Josefson, 1985; and Thoresen, 1993). One of the key concepts that has developed through this work is the notion of a caring rationality as opposed to a technical rationality. The similarities between a gardening attitude and a caring rationality are not taken up here but they are worth further theoretical investigation.

3 Situatedness is described by Suchman (1987) with respect to how we cope with computers, and by Lave and Wenger (1991) with respect to learning in the workplace as intrinsic to practical problem-solving. 